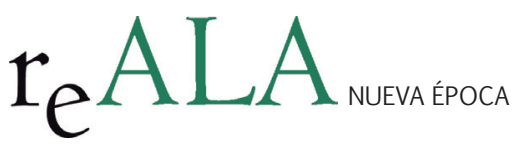

REALA, n 3, enero-junio 2015 ISSN: 1989-8975

DOI: http://dx.doi.org/10.24965/reala.voi3.10248

\title{
La importancia de la evaluación en las Políticas Públicas Españolas: una aproximación histórica basada en el método biográfico.
}

\author{
Rubén Arriazu Muñoz \\ Universidad de Extremadura \\ rarriazu@unex.es
}

\section{Resumen}

El siguiente artículo aborda de manera retrospectiva la forma en la que la cultura de la evaluación ha ido progresivamente incorporándose al ámbito de las Políticas Públicas Españolas durante las últimas tres décadas. A día de hoy, la evaluación, entendida como procedimiento de investigación autónomo, sistemático, transversal y generalizado, es un proceso que permea en la mayor parte de las instituciones públicas. Evaluación, calidad y excelencia son términos incardinados al diseño de los actuales planes, programas y proyectos de la Administración. Todo es susceptible de ser evaluado; sin embargo, llegado este momento cabe preguntarse: ‘Cómo se ha fraguado este proceso? o dicho de otro modo, ¿Cómo ha sido la historia de la evaluación en España en el ámbito de las Políticas Públicas?, ¿Cómo y por qué emerge esta nueva forma de indagación?

Para dar respuesta y sentido a estas cuestiones, se presenta una explicación longitudinal basada en el método biográfico, concretamente, en los relatos de cinco profesionales con una amplia y reconocida trayectoria profesional evaluadora dentro y fuera de España.1 El propósito es conformar una visión histórica socialmente compartida que describa la evolución de la cultura por evaluar en las Políticas Públicas Españolas partiendo de las vivencias individuales de cada profesional y siendo el testimonio individual, la base para configurar el marco general en el cual se circunscribe la historia desde abajo.

Palabras Clave

Evaluación, Políticas Públicas en España, Método Biográfico.

\section{The evaluation process in the Spanish Public Policy: a historical approach based on the biographical method}

\section{Abstract}

This paper presents a retrospective explanation as to how evaluation culture has been incorporated in the Public Administration sphere in the last three decades in Spain. Nowadays, evaluation processes are considered a systematic procedure implemented in the majority of public institutions. Evaluation, quality and excellence are relevant concepts embeddedness with the design of current plans, programs and projects. All issues within public institutions are likely to be evaluated but nevertheless, before this actually happens it is necessary to reflect: What has been the evaluation history in Spain?, How and why does it emerge and consolidate in the Public Administration this way of social research?

I address the following questions with a longitudinal description of the evaluation process in the Public Administration based on the biographical method. Specifically, it has enjoyed the narrative of five professionals with a broad and recognized evaluator career inside and outside Spain. The aim is to conform a historical approach that represents the evolution of the evaluation culture in the Spanish Public Administration, analyzing and linking the individual testimonies of professional evaluators to the specific characteristics of particular social context.

\section{Keywords}

Evaluation, biographical method, politics public in Spain.

\footnotetext{
1 Véanse las referencias y perfiles de cada profesional entrevistado al final del texto.
} 
“Las sociedades que se han industrializado advierten imprevistamente la necesidad de echar una mirada a sus espaldas, de descubrir sus raíces."

Franco Ferrarotti (1990:20)

\section{INTRODUCCIÓN: LA METODOLOGÍA BIOGRÁFICA COMO ELEMENTO PARA LA EXPLICACIÓN HISTÓRICA}

Los diferentes métodos de investigación social que vertebran el análisis de los fenómenos sociales son constructos teóricos que definen los caminos y formas específicas de examinar la realidad a partir de una base epistemológica y paradigmática consensuada. Dentro del método biográfico existe una familia de términos que se combinan para formar lo que se denomina como metodología biográfica (Denzil, 1989:13). En él tienen cabida distintas fuentes desde donde obtener la información, como por ejemplo, los “documentos personales", "cartas", “diarios", "historias de vida”, "biogramas" y "relatos” (Sánchez Valle, 1994a:119) que a su vez, se subdividen en distintas tipologías, como por ejemplo, el relato único, los relatos cruzados y/o los relatos paralelos (Pujadas, 1992: 52-57; López-Barajas, 1996:207) dependiendo de la forma, función y uso que se quiera hacer de la información.

Esta amalgama de elementos, posibilitan el acceso a dimensiones particulares de la vida del individuo, que ineludiblemente están conectadas al contexto social en el cual interactúa. El cúmulo de información, que Ferrarotti denomina como "caldo social", constituye la verdadera esencia informacional para la metodología biográfica, puesto que en ella recae su verdadero potencial para explicar, tanto lo relativo a la persona, como a la estructura social de la cual forma parte. Ejemplos de obras representativas que explican esta linealidad se encuentra en clásicos como "El Campesino Polaco" (Thomas y Znaniecki, 1918) de la Escuela de Chicago o "Los hijos de Sánchez” (Lewis, 1961). En el caso español, destacar las publicaciones de Francisco Marsal entre 1969-1979 o, más recientemente, estudios dedicados al análisis biográfico de la guerra y posguerra Civil Española como el caso “La Memoria Inquieta" (Pinillas de las Heras, 1996) o el "El final del franquismo" (Amando de Miguel, 2003).

La aplicación del método biográfico como soporte explicativo de la historia social compartida es una idea arraigada y progresivamente asumida por un determinado grupo de investigadores sociales a lo largo de los años. Ya en 1833, Dilthey proclamaba la importancia de las biografías para la comprensión cabal del hecho histórico (Sarabia, 1986:208). Thompson, Bertaux, Plummer, Ferrarotti y Catani, en Europa o Pujadas, Marinas, Santamaría, de Miguel, Oltra y Valles en España, son algunos de los nombres más significativos que han contribuido al desarrollo teórico y empírico de este modo de indagación sociológica. Este planteamiento no ha estado exento de polémica en la comunidad científica ya que ha supuesto aceptar y reformular la manera ortodoxa de abordar y explicar la historia de los fenómenos sociales establecida hasta el momento. Proyectar y conformar una "historia desde abajo" (Ferrarotti, 1990:89) ha implicado reconstruir la experiencia histórica en su variada dialéctica "El proletariado es el sujeto de la historia" (Pinilla de las Heras, 1980:183). Desde estos postulados, las historias de vida, se entienden como un medio de escucha de la cotidianidad (Ferrarotti, 1990:98) y como un modo privilegiado de crítica y desmitificación de la macrohistória, idea que ha sido sustentada y, posteriormente desarrollada, por autores como Burke (1993), Levy (1993) o Sharpe (1993) entre otros².

Profundizando sobre las cuestiones vinculadas al escepticismo y validez del método biográfico, Pujadas afirma que "el mayor de los peligros en la utilización de los relatos de vida es la fetichización del método biográfico; es decir, pensar que con uno o varios buenos relatos ya tenemos toda la información y todas las evidencias necesarias para pasar a un buen análisis y llegar a conclusiones válidas sobre un determinado problema social" (Pujadas, 1992:46). Entonces, ¿Hasta qué punto es representativo el análisis de las biografías para describir un contexto social compartido? La legitimidad y verosimilitud del método biográfico es un tema muy extenso que escapa de la pretensión en estas líneas. Sin embargo, bastará asumir la propuesta metodológica de Daniel Bertaux para justificarlo: "[... ] En investigaciones cuya concepción inicial permite la totalización de elementos de conocimiento de las relaciones socioestructurales aportados por cada relato de vida, la aparición del fenómeno de saturación me parece que es el que fundamenta la validez de la perspectiva biográfica” (Bertaux, 1993:156). Dicho de otro modo, la recurrencia/repetición en la percepción de un mismo objeto en diferentes testimonios, genera lo que Bertaux define como "saturación discursiva" (Bertaux, 2005)3. Ello, unido a un buen diseño de los perfiles biográficos,

2 La historia desde abajo se entiende como aquella que resulta de los relatos, biografías y testimonios de la gente "de a pie". Es una historia basada en lo cotidiano que se complementa con el imaginario de la macro-historia centrada más en la historia de las grandes personalidades.

3 Bertaux en el capítulo VII de su obra "Los relatos de vida. Perspectiva etnosociológica" (2005) introduce un nuevo elemento de verificación añadido a la saturación discursiva centrado en la transparencia del informe de resultados. En concreto, postula la necesidad de registrar sistemáticamente aquellos elementos distorsionantes que supongan "pistas falsas o erróneas" para el investigador. 
constituye actualmente los dos pilares básicos sobre los que fundamentar y justificar el uso del método biográfico en la investigación social.

Asumiendo estas directrices, es momento ahora de ejemplificar y aplicarlas a un caso concreto. Partiendo de que "toda obra de creación es la representación simbólica, en forma de obra cultural, de una red causal de factores subjetivos y objetivos, personales y colectivos" (Oltra et al, 2006:2), se presenta a continuación una reconstrucción histórico-social de lo que ha sido el proceso de evaluación de las Políticas Públicas Españolas. Como ya se ha señalado, la idea de evaluar en España es un procedimiento relativamente reciente que ha sido incorporado de manera progresiva en los planes, programas y proyectos del ámbito público. La pregunta ahora es: ¿Por qué surge esta preocupación por evaluar?, ¿Cómo se ha generalizado el proceso de evaluación? Para explicar este fenómeno se parte de un posicionamiento bidimensional que contempla: por un lado y desde un nivel macro, el entorno socio-político y los condicionantes sociales y, por otro y atendiendo a un nivel micro, los testimonios de cinco profesionales que han vivenciado en primera persona la evolución de dicha experiencia. La idea central es hacer converger los relatos de las experiencias personales hacia un punto central de interés para el investigador, en este caso la evaluación, del que todos los sujetos han sido protagonistas, observadores y relatores al mismo tiempo.

\section{LA EVALUACIÓN DE POLÍTICAS PÚBLICAS EN ESPAÑA DESDE UN PRISMA BIOGRÁFICO}

La evaluación entendida como "práctica de indagación analítica circunscrita dentro del ámbito de la investigación social aplicada” (Aguilar y Ander-Egg, 1992:18), cuenta con un escueto pero interesante bagaje histórico dentro del contexto español. Desde sus primeras prácticas hasta la actualidad, el proceso de evaluación ha ido desarrollando y asumiendo un progresivo rango de independencia que ha configurado su propia concepción interna ${ }^{4}$ y externa 5 .

Teniendo en cuenta el conjunto de factores sociológicos más representativos que impulsaron el proceso de evaluación en España a partir de la década de 1980, se enumera la secuencia cronológica que conforma la evolución de la evaluación en el contexto de las Políticas Públicas Españolas a partir de los siguientes periodos: 1) Génesis: primeras prácticas y la influencia anglosajona (1982-1986), 2) Una incentivación básica: la inclusión en Europa. (1986-1999), 3) Consolidación e institucionalización del proceso (2000 - ...).

CUADRO I: RESUMEN ETAPAS DE LA EVALUACIÓN DE POLÍTICAS PÚBLICAS EN ESPAÑA

\begin{tabular}{|c|c|c|c|c|c|}
\hline $\begin{array}{c}\text { ETAPAS DE LA EVALUACION DE } \\
\text { POLITICAS PUBLICAS EN } \\
\text { ESPANA }\end{array}$ & PERTODO & $\begin{array}{c}\text { CONTEXTO } \\
\text { SOCIOPOLITICO }\end{array}$ & $\begin{array}{l}\text { CARACTERISTICAS } \\
\text { DEL MODELO DE } \\
\text { EVALUACION }\end{array}$ & $\begin{array}{l}\text { CRITERIOS DE } \\
\text { EVALUACION }\end{array}$ & $\begin{array}{c}\text { AMBITOS DE } \\
\text { IMPLEMIENTACION }\end{array}$ \\
\hline 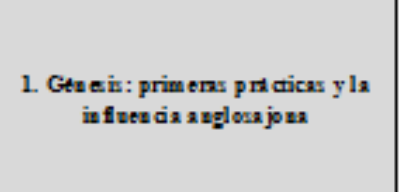 & $1982-1995$ & 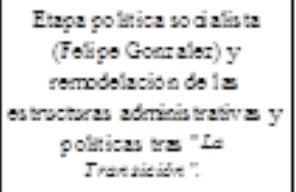 & 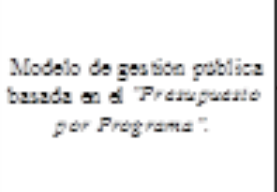 & 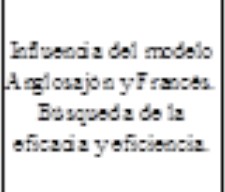 & 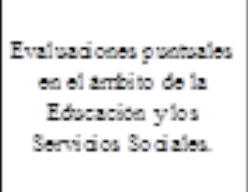 \\
\hline $\begin{array}{l}\text { 2. Una incen fivacion basica: la } \\
\text { ischasion on Furopa }\end{array}$ & $1956-1999$ & 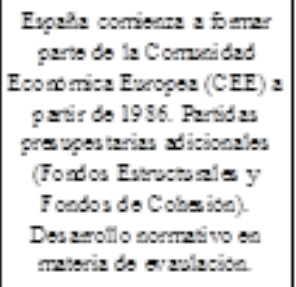 & 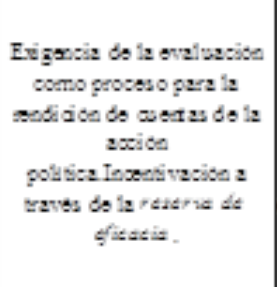 & 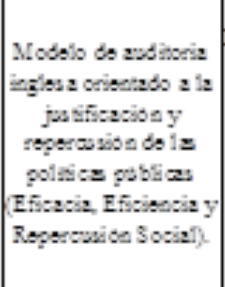 & 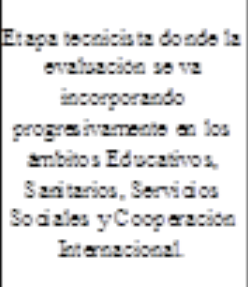 \\
\hline $\begin{array}{l}\text { 3. Consolid acion o ins fim cionslinacion } \\
\text { del proceso }\end{array}$ & $2000-\ldots$ & 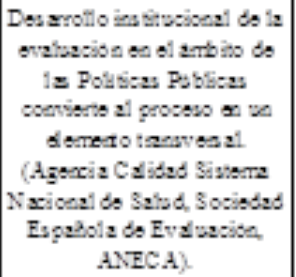 & 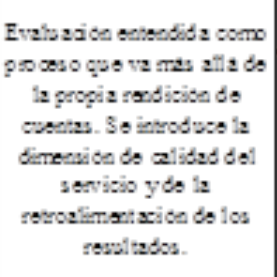 & 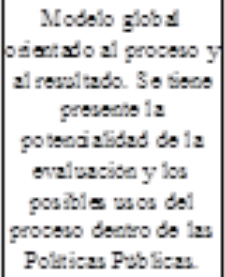 & 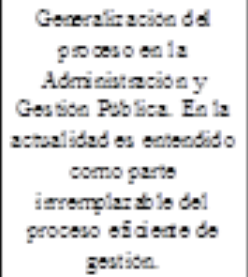 \\
\hline
\end{tabular}

Fuente: Elaboración propia

4 La concepción interna del proceso de evaluación hace referencia a los planteamientos metodológicos y el diseño con los que se decide evaluar.

5 La concepción externa hace referencia a la relevancia social del procedimiento, es decir, a la legitimidad de la evaluación como forma autónoma de proceder y a la justificación y funcionalidad atribuida al proceso desde las instancias públicas y/o políticas. 


\subsection{Génesis: primeras prácticas y la influencia anglosajona (1982-1986)}

Tras momentos de crisis y crispación política, en1982 el Partido Socialista Obrero Español (PSOE) se alzó como vencedor de las Elecciones Generales a la Presidencia del Gobierno en España (Tusell, 1997:57). Ello se tradujo en la aplicación de nuevas medidas, competencias y responsabilidades que impulsaron el proceso de evaluación en las Políticas Públicas. Una de las más representativas, resultó ser el modelo de gestión pública conocido como el "Presupuesto por Programas".

El “Presupuesto por Programas" - versión adaptada del "Rationalisation des Choix Budgétaires" francés (Ballart, 1992:63) cuyos principios inspiradores se ciñeron, en ambos casos, a los planteamientos del modelo de gestión estadounidense PPBS $^{6}$ asumió el reto de reconfigurar la estructura organizativa y burocrática del país a partir de una serie de criterios racionales. Sin embargo, esta iniciativa no obtuvo los resultados esperados. Las principales deficiencias del "Presupuesto por Programas" se encontraron en su trasfondo metodológico, concretamente, en el diseño, definición de objetivos y operacionalización de indicadores para evaluar la eficacia de los servicios (Zapico, 1989:194-95; Ballart, 1992:64). En palabras de Subirats: "El balance de todo ello es la preocupación por el procedimiento más que la preocupación por el resultado de la acción administrativa” (Subirats 1989:24; Ballart, 1992:64).

Así pues, con la puesta en marcha de este modelo de gestión surge, si se quiere denominar así, el primer atisbo de la conciencia evaluadora dentro de la Administración Pública Española. Ahora bien, como dejan entrever los argumentos de Zapico y Subirats, estos inicios no fueron muy alentadores en la praxis puesto que presentaron graves problemas de fondo. Este planteamiento es igualmente compartido por Xavier Ballart, que a este respecto incorpora una visón más concluyente afirmando que "[... ] la estructura administrativa existente y los mecanismos de decisión [del Presupuesto por Programas] no favorecieron a la evaluación” (Ballart, 1992:65).

En cualquiera de los casos, lo importante aquí no será tanto analizar los problemas que caracterizaron a estas primeras actuaciones, sino señalar que los modelos de gestión públicos basados en criterios de eficacia y eficiencia fueron las primeras propuestas evaluativas tomadas de modelos precedentes ya implementados en Francia y Estados Unidos.

El contexto de cambio promovido desde el ámbito ministerial se complementa con la percepción biográfica de los primeros evaluadores. En esta etapa inicial, es un periodo donde las evaluaciones se suceden de forma puntual y en áreas muy concretas como Educación o Servicios Sociales. Resulta interesante examinar la importancia y naturaleza que se tenía del proceso. ¿Qué se entendía por evaluación?, ¿Cómo y por qué se llevaba a cabo? Según relata uno de los primeros expertos españoles en la materia, el concepto de evaluación en España en la década de los 80 era difuso, es decir, la gente identificaba el concepto, no así su verdadera condición.

“[...] en los inicios de la evaluación la gente hablaba de evaluación pero no sabía muy bien qué era, es decir, las palabras se ponen de moda, ahora está de moda calidad. Dentro de poco desaparecerá y se pondrá otra. Entonces lo de investigar ya no estaba tan de moda. Evaluación parecía que era más correcto. Entonces empezaron a surgir pequeñas presiones para que se evaluaran las cosas. En el campo que yo empecé, en las Intervenciones Sociales, en Servicios Sociales, en Drogas, pues igual ¿no? Esa presión surgía de una manera difusa, o bien, eran los Directivos, o bien, eran los Consejos Sociales, o bien eran las ONG, o bien era la oposición en el Ayuntamiento... "porque esto hay que evaluarlo". Pero era evidente que estas presiones eran de tipo político pero que reflejaban la idea de que tenemos que conocer qué pasa y tenemos que tener un cierto control. Esa era la idea que había detrás ¿no? Luego siempre se decía que era para mejorar, pero la idea era una cuestión que estaba así."

Pese al desconcierto terminológico, el relato pone de manifiesto un hecho clave que evidencia y vincula la función de los procesos evaluativos con la justificación o rendir cuentas -“accountability”- (Chelimsky, 1997:58), es decir, se decide evaluar para conocer e informar qué está pasando. Otro elemento importante que paralelamente impulsó la cultura por evaluar en España fue "el vínculo político". Esta dimensión asociada al proceso de evaluación desde sus inicios, fue descrita en 1975 por Carol Weiss, quién identificó y teorizó sobre la relación latente entre evaluación y política7. La evaluación pasó así a formar parte del engranaje político en la medida que supuso un

6 El PPBS -“Programming Planning and Budgeting System”- es un modelo programático que desarrolló el Departamento de Defensa de los Estados Unidos durante el periodo de la II Guerra Mundial con objeto de racionalizar el presupuesto y gasto destinado a la gestión de programas militares.

7 La relación entre el político y el científico es funcional en la media que representan intereses recíprocos. Véase también Weber, M. (1981) El Político y el Científico. Madrid, Alianza. 
mecanismo investigador y/o justificador de los programas impulsados a través de Políticas Públicas. Esta anexión interdependiente constituye una de las claves para entender hoy la importancia que se le atribuye a este proceso.

Trasladando este planteamiento al ámbito español, resulta significativo observar el funcionamiento interno bajo el que se fraguó una de las primeras evaluaciones de gran envergadura en el ámbito educativo. El entrevistado número 2, en calidad de director del proyecto, explica el modo en que se desarrolló la evaluación de la Reforma de las Enseñanzas Medias en España.

“En el año 82 cuando el PSOE gana las elecciones quieren hacer una reforma en las enseñanzas medias, y yo trabajo en el Centro de Investigación y Documentación Educativa. Entonces yo mismo por mi parte tomo la iniciativa y digo que antes de ponerlo en marcha y dar como definitiva una reforma tienen que evaluarla. $Y$ entonces, previa petición por carta, se me encarga hacer esa evaluación de las reformas de las enseñanzas medias [...] empiezo en el año 83-84 y estoy evaluando hasta el año 92 [...] esto es en el Ministerio de Educación y Ciencia."

La decisión y predisposición para llevar a cabo este proyecto, pionero en España, ${ }^{8}$ no surge de manera espontánea sino que tiene su origen en la trayectoria y experiencia vital del propio entrevistado quien mantenía una estrecha vinculación con el Educational Testing Service (ETS) americano, organismo, al que se trasladó en varias ocasiones y con el que colaboró en varios proyectos internacionales conjuntos.

En lo que al trasfondo metodológico respecta, la evaluación de la Reforma de las Enseñanzas Medias se diseñó partiendo del paradigma cuantitativo con grupos cuasi-experimentales y de control, 9 modelo característico y validado en la mayor parte de las evaluaciones realizadas en Estados Unidos en aquel momento.

"Yo hacía una evaluación cuantitativa para el Ministerio de Educación. [...] todas las publicaciones que hace el Educational Testing Service (ETS) es evaluación de orientación cuantitativa fundamentalmente."

En resumen, los inicios de la cultura de la evaluación en España se circunscriben a un contexto sociopolítico frágil que emerge tras el periodo conocido como "La Transición". La asunción de un nuevo modelo de gestión pública basada en criterios racionales de eficacia y eficiencia fue uno de los primeros precedentes que impulsan sin demasiado éxito la evaluación en la esfera de las Políticas Públicas. Es un periodo donde el desarrollo de evaluaciones se implementaba de manera puntual y en contextos muy específicos tomando como referente los diseños metodológicos anglosajones. No existe una clara predisposición por evaluar. El testimonio del entrevistado número 3 no deja lugar a dudas cuando afirma.

“[...] es una evaluación que yo hago en el año 1988 con lo cual realmente no existe una demanda ni una necesidad de evaluación [...] tuve que hacer todo un trabajo de persuasión y sensibilización de que la evaluación servía para algo."

El principal reto de la evaluación en estos primeros años era demostrar su funcionalidad y potencialidad, es decir, introducir una serie de prácticas orientadas a la mejora y perfeccionamiento de un sistema de políticas públicas en periodo de consolidación. En el escenario socio-político coyuntural de la época, el proceso evaluativo era una actividad incipiente más vinculada con la gestión y burocratización de las políticas propias públicas que con la mera detección y mejora de los planes, programas y proyectos evaluados. Es una práctica indagatoria exportada y eso precisó de un periodo de persuasión y sensibilización por parte de la primera generación de los evaluadores.

\subsection{Una incentivación básica: la inclusión en Europa (1986-1999)}

El 1 de enero de 1986 España pasó a formar parte de los Estados Miembros de la Comunidad Económica Europea (CEE), desde 1992, Unión Europea. Ello derivó en una nueva situación económica para el país al convertirse en parte de un robusto enclave económico. La CEE supuso un beneficio para España en la medida que dispuso de una serie de partidas económicas a través de los Fondos Estructurales y los Fondos de Cohesión (Carrasco, 1999:29) que hicieron posible el diseño, la implementación, y consecuentemente, la evaluación de un conjunto de programas y proyectos de carácter público.

8 Salvando las distancias, una de las primeras evaluaciones realizadas en EE.UU fue también en el ámbito educativo - "Eight Year Study"- . Su publicación se encuentra disponible en: Smith, E. R. \& Tyler, R. W. (1942). Appraising and recording student progress. Nueva York: Harper \& Row. McNally.

9 Véase Cook, T. D. \& Campbell, D. T. (1979). Quasi-experimentation: Design and Analysis Issues for Field Settings. Chicago: Rand 
Durante este periodo próspero, la Unión Europea concedió grandes sumas de capital para equiparar el nivel de bienestar de los ciudadanos europeos. Como contrapartida, y como parece lógico, demandó conocer la eficacia, eficiencia y repercusión de dichas inversiones. El resultado, como reconoció Carmen Vélez en su artículo, es una creciente preocupación por evaluar las Políticas Públicas.

“Desde el periodo de actuación 1988/93, 1994/99 hasta el actual Marco Comunitario de Apoyo (2000/06) los reglamentos comunitarios han endurecido sus exigencias en materia de evaluación." (2001:3)

Bajo esta lógica, la política de la Unión Europea hizo un llamamiento a la responsabilidad y buenas prácticas en la evaluación de los programas financiados con los Fondos Europeos. Para ello, dispuso de instrumentos específicos como la "reserva de eficacia" para garantizar e incentivar el desarrollo de este proceso.

“La reserva de eficacia, esto es, una parte de los créditos asignados a cada Estado Miembro (el 4\%) se ha dejado en reserva hasta el 2003 para ser distribuida a los programas que hayan obtenido mejor rendimiento. La apreciación del rendimiento se realizará por medio de indicadores de seguimiento que reflejen la eficacia, la gestión, y la ejecución financiera. Se trata de una importante motivación para que los responsables de los programas se aseguren de que los fondos públicos se utilicen de forma eficaz y rentable." (2001:5)

La cultura por evaluar comienza a cobrar importancia en las Políticas Públicas Europeas y Españolas en la medida que supone un procedimiento autónomo que contribuye a justificar y/o rendir cuentas del "buen o mal" funcionamiento de la política. La ejecución de la evaluación supuso activar un mecanismo de incentivos, concretado en la dotación de la reserva de eficacia, que dio continuidad a los planes, programas y proyectos eficientes preexistentes. La evaluación en este punto comenzó a entenderse como la acción indagatoria válida para legitimar la gestión eficaz, eficiente y transparente de las Políticas Públicas.

Dentro de la Administración Pública Española el planteamiento europeísta generó una creciente y efectiva demanda de información evaluativa en los distintos niveles de Gobierno (Ballart, 1992:65). Se articularon procedimientos encaminados al análisis de la eficacia y gestión de los distintos ámbitos ministeriales. Sin embargo, el resultado, según reconoce el autor, siguió más la línea de la gestión de las auditorías inglesas que las evaluaciones de programas o servicios.

La línea tecnicista descrita por Ballart converge con la percepción obtenida desde el ámbito biográfico, donde igualmente se constata la rigurosidad de la práctica.

“En los 90...la palabra evaluación existía, porque estaba en el discurso, la gente hablaba de evaluación y además estaba muy connotada de ser técnico, de ser riguroso y que siempre había que evaluar".

Sin embargo, esta idea primigenia y tecnocrática va diluyéndose a lo largo de los años en favor de una concepción más amplia de la evaluación. Este procedimiento emerge como mecanismo para identificar y rendir cuentas, pero su funcionalidad trasciende a un plano más efectivo en el que tiene también cabida la retroalimentación y/o mejora de las deficiencias detectadas en las Políticas Públicas. Esta particularidad afianzó el vínculo entre el proceso de evaluación y las acciones impulsadas desde el ámbito de la Administración Pública, hecho que cristalizó en la creación de las primeras Sociedades específicas en materia de Evaluación en Europa. ${ }^{10}$ En el caso de España, la importancia del proceso de evaluación comienza paulatinamente con la inclusión de nuevas directrices legislativas y la aparición de los primeros centros específicos en materia de evaluación. Haciendo una breve recapitulación por los ámbitos donde se asumen un mayor compromiso conviene mencionar:

La evaluación en el Ámbito Educativo: La Ley Orgánica de Ordenación General del Sistema Educativo (LOGSE) del 3 de octubre de 1990 supone el punto de partida de un proceso de profunda reforma de la educación en España. El artículo 62 de la citada Ley insta explícitamente en su artículo 62 a la creación del Instituto Nacional de Calidad y Evaluación (INCE), que tres años más tarde y en base al Real Decreto 928/1993, comenzó a desarrollar sus acciones y competencias específicas en el ámbito de la evaluación educativa.

10 Una de las instituciones más destacadas en Europa es la Sociedad Europea de Evaluación -fundada en La Haya en 1994-, que contó durante los primeros años con la colaboración activa de D. ${ }^{a}$ Rocío Fernández Ballesteros, discípula del estadounidense Cronbach, y una de las pioneras evaluadoras españolas en el área de las Políticas Públicas. En la actualidad, María Bustelo también ha sido una figura representativa de esta institución ostentando el cargo de presidenta durante los años 2012 y 2013. 
La evaluación en el Ámbito Sanitario: El crecimiento imparable del Gasto Público Sanitario en España a principios de 1990 derivó en la necesidad de racionalizar el sistema y controlar sus costes. En 1991 el entonces Ministro Julián García Vargas propuso una Comisión de Expertos en la materia y estableció un paquete de medidas encaminadas a mitigar y controlar los efectos del gasto. Una de las propuestas más representativas fue la constatada en el Real Decreto 1415/1994 bajo el cual germina la Agencia de Evaluación de Tecnologías Sanitarias, encuadrada dentro del Instituto de Salud Carlos III. Su objetivo, ofrecer un conjunto de valoraciones objetivas sobre los impactos sanitario, social, ético y organizativo.

La evaluación en el Marco de la Cooperación Internacional: De igual modo que en las dos áreas precedentes, la evaluación comienza a formar parte del ámbito de la Cooperación Internacional a través de la aprobación de medidas legislativas. En esta ocasión, la Ley 23/1998 de Cooperación Internacional para el Desarrollo, en su artículo 19, instó a la Secretaría de Estado de Cooperación Internacional a ser la responsable de evaluar la política de cooperación para el desarrollo de España, así como los programas y proyectos financiados por el Estado Español sobre esta materia.

Como ha quedado patente, la inclusión en la CEE supuso un importante revulsivo que contribuyó a fomentar la evaluación en España. La implementación de una serie de medidas que demandaban conocer la eficacia y eficiencia de los programas financiados con Fondos Europeos, generó intrínsecamente mecanismos e instrumentos de evaluación que fueron incorporados a las Políticas Públicas Españolas. Y es a partir de entonces, y en base a su doble funcionalidad -rendir cuentas y retroalimentar-, cuando la evaluación cobró fuerza y sentido a través de su mención explícita en las disposiciones legislativas. Se abre así un nuevo panorama para la evaluación en la esfera de las Políticas Públicas. La necesidad de justificar presupuestariamente los resultados de las iniciativas políticas ante organismos internacionales cristaliza en una generalización del proceso a todos los niveles. En concreto, el personal técnico de la Administración Pública fue uno de los principales responsables de incluir esta práctica como parte esencial de su trabajo. Es un periodo donde prima la funcionalidad de las actuaciones políticas; sin embargo, es importante señalar que se trata de un periodo todavía gestacional donde la evaluación tiene una dimensión reduccionista muy centrada en la valoración o efecto de los proyectos o políticas. Será en años posteriores, y al amparo del dinamismo social de las políticas públicas, cuando nuevamente se reformule el sentido y la importancia con el que entendemos hoy el proceso de evaluación en España.

\subsection{Consolidación e Institucionalización del proceso (2000-...)}

La entrada en el Siglo XXI supone un periodo de consagración para la evaluación en España. El desarrollo normativo de la etapa anterior trasciende a todos los niveles y ámbitos de las Políticas Públicas. El concepto y la idea de evaluar se ha convertido en ejes transversales del diseño de cualquier política y/o programa público y las acciones evaluativas han evolucionado progresivamente hacia un plano de normalización. Esta situación queda patente en los relatos de dos de los profesionales entrevistados que han tenido la ocasión de vivir "el tránsito evaluativo español" hasta el momento actual.

“[...] El panorama y el contexto ha cambiado radicalmente, ahora la idea de que hay que evaluar está mucho más incorporada. En poco tiempo se ha institucionalizado la función de evaluación, ahora tenemos una Sociedad Española de Evaluación que no había."

"[...] Si tú les preguntas qué es evaluar ya casi todos hablan de criterios de valores de diferentes tipos de evaluación. Saben que hay evaluaciones de resultados etcétera, etcétera, o sea, que la gente está muchísimo más formada, y a la vez, hay muchísima más gente en el campo de la evaluación, pues implica que se hacen muchísimas más evaluaciones."

Como ya se anticipa, la institucionalización de la evaluación en el ámbito de las Políticas Públicas constituye el último estadio para la consolidación del proceso. Si bien con la mención legislativa de la década de 1990 emergen las primeras instituciones específicas, en este periodo la dimensión institucional adquiere pretensiones y tintes más universalistas. De acuerdo al orden cronológico con el que han sido creadas, se pasa a enumerar la infraestructura más representativa del área de evaluación de las Políticas Públicas Españolas:

1. Sociedad Española de Evaluación (SEE): Fundada en el año 2001 con el apoyo de la Sociedad Europea de Evaluación, es constituida con el objetivo de estimular y fomentar los procesos de evaluación en España y especialmente, en la Administración Pública.

2. Agencia Nacional de Evaluación de la Calidad y Acreditación (ANECA): Institución que comienza su funcionamiento en 2002 de acuerdo a lo establecido en la Ley Orgánica de Universidades 6/2001 (LOU) y 
al amparo de las recomendaciones del Comunicado de Berlín en materia de Espacio Europeo de Educación Superior (EEES). Sus funciones y competencias se ciñen a la coordinación y dinamización de las políticas de evaluación y gestión de la calidad para el logro de la excelencia en las universidades españolas.

3. Agencia de Calidad del Sistema Nacional de Salud: Con la aprobación de la Ley 16/2003 de cohesión y calidad del Sistema Nacional de Salud, nace la Agencia de Calidad del Sistema Nacional de Salud como organismo dependiente del Ministerio de Sanidad y Consumo. Esta institución, que suprime a la Dirección General de Planificación Sanitaria, asume el compromiso de garantizar la excelencia y cobertura de los servicios públicos sanitarios en España.

4. Agencia Estatal de Evaluación: En 2004 las instancias gubernativas comenzaron a trabajar en el diseño de la Agencia Estatal de Evaluación de Políticas Públicas y de Calidad de los Servicios. A día de hoy este proyecto es una realidad. El nuevo organismo regulado por la Ley 28/2006 es dependiente directo del Ministerio de Administraciones Públicas. Su cometido prioritario es el fomento de evaluaciones de políticas, planes, programas y proyectos cuya gestión corresponde a la Administración General del Estado.

Como se puede observar, el desarrollo institucional lleva implícito una mayor preocupación por la especialización del proceso de evaluación y la mejora de la calidad, hecho que ha cristalizado en una mayor intensidad del desarrollo de sus prácticas. Según reconoce el Ministerio de Administraciones Públicas, en la actualidad la actividad evaluadora se extiende en áreas como:

- Evaluaciones centradas en Políticas Sociales.

- Evaluación de la Asignación y Empleo de los Fondos Comunitarios.

- Evaluación de la Calidad de los Servicios.

- La labor de Inspección de Servicios realizada por los Ministerios.

Todas estas dimensiones analíticas constituyen los nuevos horizontes para la evaluación de las Políticas Públicas en España, y será ahora, en un momento de consolidación institucional, cuando la conciencia por evaluar se incorpore más si cabe como parte esencial que defina las acciones público-políticas haciendo progresivamente extensible su potencial a otros ámbitos que competen a la Administración del Estado. El reto de futuro no se ocupa ya de la evaluación final, de los resultados del proyecto o del beneficio que reporte un plan o política determinada si no que trata ir más allá. La institucionalización y la consolidación del proceso de evaluación en las Políticas Públicas Españolas abre un nuevo abanico en sus concepciones a la hora de plantear la evaluación. Existe un entendimiento socialmente aceptado que va más allá de la evaluación final como único recurso para rendir cuentas. Es más, la connotación del término "calidad" en el ámbito de las Políticas Públicas, lleva implícitamente una reconceptualización de la idea de evaluación hacia un enfoque multidimensional que afecta tanto a los momentos en que se lleva a cabo su práctica como a los agentes a los que va dirigido. El desafío futuro de la evaluación deberá ser de carácter adaptativo. En un modelo de Estado en continuo dinamismo, sus instrumentos indagatorios deben ser cada vez más flexibles y adaptados tanto para identificar fortalezas y debilidades como para plantear recursos y soluciones en el corto y medio plazo.

\section{CONCLUSIONES}

A la luz de los planteamientos expuestos, la cultura de evaluación de las Políticas Públicas Españolas cuenta en la actualidad con una trayectoria histórica de más de tres décadas. Sin lugar a duda, uno de los aspectos más significativos, que impulsó y determinó el lugar que ocupa este procedimiento sistemático es el vínculo político. La correspondencia entre "evaluación y política" comenzó siendo un elemento subyacente que progresivamente adquiere una mayor relevancia a partir de su propia funcionalidad. La evaluación en España, al igual que en el resto de contextos, se ha consolidado en sus inicios como mecanismo justificador para "rendir cuentas", ahora bien, el "rendir cuentas" entraña por sí mismo, una serie de intereses manifiestos y latentes para las figuras intervinientes -evaluador-gestor/político- susceptibles de analizar e interpretar con material empírico en un nuevo texto.

En consonancia con este planteamiento, resulta igualmente destacable la incidencia que ha tenido, y tiene, el marco europeo en el desarrollo de la cultura de evaluación en España y en el resto de Estados Miembros. Nuevamente, la necesidad de informar, en este caso sobre el funcionamiento de los proyectos impulsados con dotaciones/fondos europeos, ha estimulado la práctica evaluadora en toda Europa. En el caso español, la legitimidad de la evaluación como proceso vino acompañada de un marco normativo específico que incentivó la práctica, y posteriormente, impulsó la creación de las instituciones específicas en materia de evaluación. La tarea y el reto futuro es saber qué uso corresponde a este tipo de instituciones evaluadoras. Como señala Subirats: "La utilización de los resultados de toda evaluación supone pues, en la mayoría de los casos, un juicio de valor 
de carácter político" (Subirats, 2008: 223). Sobre esta cuestión, Alvira et al, ponen de manifiesto la extensibilidad de la práctica evaluativa en España afirmando que "actualmente, todas las organizaciones y entidades públicas y privadas, incluidas las universidades, establecen sistemas de evaluación para demostrar su credibilidad hacia el exterior, y para producir información utilizable en la gestión de las mismas" (Alvira, 2014: 23). Sin embargo, y como hemos apuntado anteriormente, el desafío de la evaluación de las Políticas Públicas Españolas se encuentra en su capacidad adaptativa, es decir, no es recomendable imponer modelos de evaluación estandarizados, sistemáticos y generalizados para contextos, situaciones y realidades sociales completamente heterogéneas. Esta disyuntiva es puesta de manifiesto por Subirats (2005), cuando afirma que las evaluaciones de las políticas sociales tienen que estar sujetas a un planteamiento diferente donde, no se valore tanto la eficacia y eficiencia interna, si no que se abogue por un análisis de la calidad de las interrelaciones entre esas organizaciones públicas y la ciudadanía. Es necesario partir de las particularidades del ámbito y las personas implicadas en la evaluación ya que, como señala Gary Henry (2003), "la evaluación del programa es en sí misma una intervención social” (citado en Alvira, 2014:20) sobre el medio y sobre las personas.

Por otro lado, subrayar la potencialidad del método biográfico para la explicación de un fenómeno histórico y social. La combinación de elementos que configuran la macro-historia, como puede ser la bibliografía referente al tema, con los relatos o testimonios individuales -micro-historia- supone enlazar de una forma plausible el modo de comprender y explicar una realidad dada. Entremezclar ambas vertientes, incluir lo cotidiano en la pretensión investigadora enriquece, complementa y aporta una visión más precisa del fenómeno explicado, en este caso la evaluación de las Políticas Públicas Españolas. Resulta en cierto modo contraponer el argumento de Geertz cuando constata: "Ia rareza que supone construir textos ostensiblemente científicos a partir de experiencias claramente biográficas, que es lo que al fin y al cabo hacen los etnógrafos, queda totalmente oscurecida" (Geertz, 1989:19), y abogar por una idea donde el investigador social debe ser consciente tanto de los fenómenos sociales como de la percepción social que reporta en los sujetos que lo vivencian. En la conexión de estos dos puntos está el eje para conformar una historia social compartida, verosímil y legítima ante los ojos de la Comunidad Científica.

Finalmente, es importante aludir a la idea de "Historia" como fenómeno social dinámico y en continuo tránsito. La necesidad de comprender el presente pasa por analizar el pasado. Buena cuenta de este argumento llevan dando durante los últimos años King, Melvin, Miller y Stockdill en la revista de la Asociación Americana de Evaluación a través de su proyecto "The Oral History of Evaluation" -La Historia Oral de la Evaluación-. En él rescatan, en forma de entrevista, los testimonios de los primeros evaluadores estadounidenses aportando percepciones, vivencias y situaciones que escapan de la historia de la evaluación estadunidense manifestada hasta el momento. Así pues, será labor de todos otorgar la relevancia que corresponde a lo cotidiano en el marco de la historia a partir de ejemplos científicos. La reconstrucción y/o deconstrucción de los fenómenos sociales globales remite en última estancia a las personas, individuos o sujetos poseedores de una biografía ligada a un contexto interdependiente y en continuo tránsito, y es por esta razón, y en la combinación de ambas dimensiones -contextual-individuo-, donde encuentra sentido la explicación histórica global del fenómeno sociológico.

\section{BIBLIOGRAFÍA}

Alvira, F. et al (2014): Usos de la evaluación del profesorado universitario en España. Ediciones Institucionales $n^{\circ}$ 104. Universidad Castilla la Mancha.

Aguilar, M. J. y Ander-Egg, E. (1992): Evaluación de servicios y programas sociales, Madrid, Siglo XXI.

Ballart, X. (1992): ¿Cómo evaluar programas y servicios públicos? Aproximación sistemática y estudios de caso, Madrid, Ministerio para las Administraciones Públicas.

Bertaux, D. (1993): “La perspectiva biográfica: validez metodológica y potencialidades" en Marinas, J. M. y Santamarina, C. (ed.) La Historia oral: métodos y experiencias, Madrid, Debate, pp. 149-173.

Burke, P. (1993): Formas de hacer historia, Madrid, Alianza.

Carrasco, E. (1999): “Los Fondos Estructurales y de Cohesión. Objetivos y Realidades" en Boletín Económico de ICE, Madrid, ICE, N²633, pp. 29-37.

Chelimsky, E. y Shadish, W. R. (1997): Evaluation for the 21st Century. A Handbook, London, Sage.

Denzin, N. K. (1989): Interpretive Biography, London, Sage.

Ferrarotti, F. (1990): La historia y lo cotidiano, Barcelona, Ediciones Península.

Geertz, C . (1989): El antropólogo como autor, Barcelona, Paidós. 
López-Barajas, E. (1996): Las historias de vida y la investigación etnográfica. Fundamentos y metodología. Madrid, UNED.

MAP (2006): “Evaluación institucional”. Recurso electrónico del Fondo Documental virtual del Ministerio de Administraciones Públicas. Disponible en:

http://www.map.es/iniciativas/mejora_de_la_administracion_general_del_estado/servicio_publicos/ evaluacion_calidad/evaluacion/institucionalizacion/documentos_publicaciones.html.

Oltra, B. / Garrigós, J. I. / Mantecón, A. / Oltra Algado, C. (2004): Sociedad, vida y teoría. La Teoría Sociológica desde una perspectiva de sociología narrativa, Madrid, CIS.

Pinilla de las Heras, E. (1980): “Las clases sociales: realidad social y problema teórico" en

Marsal J.M. y Oltra, B. (ed.) Nuestra Sociedad. Introducción a la Sociología, Barcelona, Vicens, pp.151-191.

Pujadas, Muñoz, J. J. (1992): El Método biográfico: el uso de las historias de vida en ciencias sociales, Madrid, Centro de Investigaciones sociológicas.

Sánchez Valle, I. (1994): "Desarrollo de métodos cualitativos de investigación en pedagogía: el método biográfico" en López-Barajas, E. y Montoya, J.M. (ed.) La investigación etnográfica. Fundamentos y técnicas, Madrid, UNED, pp.119-143.

Sarabia, B. (1986): "Documentos personales: historias de vida" en García Ferrando, M. / Ibáñez, J. / Alvira; F. (ed.) El análisis de la realidad social, Madrid, Alianza, pp. 205-226. 58.

Sharpe, J. (1993): “La historia desde abajo” en Burke, P. (ed.) Formas de hacer historia, Madrid, Alianza, pp. 38-

Stufflebeam, D. y Shinkfield, A. (1987): Evaluación sistemática. Guía teórica y práctica. Madrid, Paidós/MEC.

Subirats, J. (1989): Análisis de políticas públicas y eficacia de la administración, Madrid, Ministerio para las Administraciones Públicas.

Subirats, J. (2005): "Catorce puntos esenciales sobre evaluación de políticas públicas con especial referencia al caso de las políticas sociales", Ekonomiaz, 60 (1):18:37

Subirats, J. et al (2008): Análisis y Gestión de Políticas Públicas, Barcelona: Ariel.

Tusell, J. (1997): “La Transición” en Historia de España Vol. 14. Enciclopedia de España, Madrid, Espasa.

Velez, C. (2001): “La Evaluación de las Políticas Públicas en España”, Recurso electrónico del Fondo Documental virtual del Ministerio de Administraciones Públicas. Disponible en: http://ioce.net/download/case_studies/la evaluacion.doc.

Weiss, C. H. (1975): Investigación evaluativa. Métodos para determinar la eficiencia de los programas de acción, México, Trillas.

Zapico, E. (1989): La modernización simbólica del presupuesto público, Oñati, IVAP.

\section{REFERENCIAS ENTREVISTADOS}

Mariano Álvaro Page ha trabajado dirigiendo el Servicio de Evaluación del Centro de Investigación y Documentación Educativa (CIDE) desde 1983 a 1992. Durante este periodo ha colaborado en diferentes evaluaciones y proyectos con el Educational Testing Service en Estados Unidos. En la actualidad es subdirector General de Estudios y Cooperación en el Instituto de la Mujer encargado de diseñar las evaluaciones de las Políticas de Género.

Francisco Alvira Martin ha sido profesor visitante en la Universidad de Michigan y la Universidad Northwestern invitado por el profesor Robert Boruch. Posee una larga y reconocida trayectoria como evaluador en el ámbito de los Servicios Sociales. Una de sus contribuciones más importantes al campo de la evaluación fue la obra publicada en 1991 "Metodología de evaluación de Programas". En la actualidad es Catedrático y Director del Departamento de Metodología de la Investigación Social en la Universidad Complutense de Madrid.

María Bustelo Ruesa ha sido profesora visitante en el Center for Instructional Research and Curriculum Evaluation en la Universidad de Illinois. Ha trabajado como evaluadora en el área de Políticas Públicas, especialmente en los ámbitos de Servicios Sociales y Educación durante gran parte de su carrera profesional. En la actualidad es Profesora Titular en el Departamento de Ciencia Política de la Universidad Complutense de Madrid y Directora del Máster en Evaluación de Programas y Políticas Públicas ofertado por esta misma universidad. 
María José Aguilar Idáñez es directora del Departamento de Servicios Sociales y Trabajo Social en la Universidad de Castilla La Mancha. Ha trabajado como evaluadora en el Programa de Cooperación y Desarrollo de Naciones Unidas desarrollando proyectos y evaluaciones en la mayor parte de países latinoamericanos. En la actualidad es directora del Instituto de Desarrollo Regional de Albacete y Catedrática de Escuela Universitaria en el área de Trabajo Social y Servicios Sociales.

Juan Andrés Ligero Lasa es profesor asociado en el Departamento de Sociología y Ciencia Política de la Universidad Carlos III de Madrid. Posee una reconocida carrera profesional como evaluador externo para la Administración Pública, especialmente en el área de Servicios Sociales. Actualmente ocupa el puesto de Co-Director del Máster Máster en Evaluación de Programas y Políticas Públicas ofertado por la Universidad Complutense de Madrid. 
